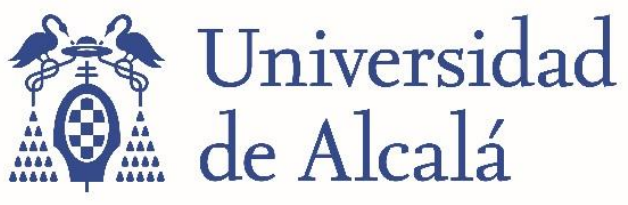

BIBLIOTECA

Document downloaded from the institutional repository of the University of Alcala: http://ebuah.uah.es/dspace/

This is a postprint version of the following published document:

Caravantes, J., Sendra, J.R., Sevilla, D. \& Villarino, C. 2018, "On the existence of birational surjective parametrizations of affine surfaces", Journal of Algebra, vol. 501, pp. 206-214

Available at http://dx.doi.org/10.1016/i.jalgebra.2017.12.028

(C) 2018 Elsevier

(Article begins on next page)

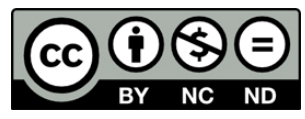

This work is licensed under a

Creative Commons Attribution-NonCommercial-NoDerivatives

4.0 International License. 


\title{
On the existence of birational surjective parametrizations of affine surfaces
}

\author{
J. Caravantes, J.R. Sendra, D. Sevilla and C. Villarino
}

December 5, 2017

\begin{abstract}
In this paper we show that not all affine rational complex surfaces can be parametrized birational and surjectively. For this purpose, we prove that, if $S$ is an affine complex surface whose projective closure is smooth, a necessary condition for $S$ to admit a birational surjective parametrization from an open subset of the affine complex plane is that the curve at infinity of $S$ must contain at least one rational component. As a consequence of this result we provide examples of affine rational surfaces that do not admit birational surjective parametrizations.
\end{abstract}

2010 Mathematics Subject Classification. Primary 14Q10, 68W30.

Keywords. Rational surface, birational parametrization, surjective parametrization.

\section{Introduction}

Some computational problems, of mathematical nature, can be approached by means of algebrogeometric techniques. In these situations, either because of the problem itself directly relates to an algebraic variety or because the problem is translated into an underlying algebraic variety, techniques from computational algebraic geometry are applied. Specially important are those cases where the associated algebraic variety is unirational, since then two different types of representations of the geometric object, namely a set of generators of its ideal or a rational parametrization of it, are available. Examples of this claim appear in some practical applications in computer geometric design (see [2], [5], [11], [16]), where the connection to algebraic geometry is direct. Other examples can be found in the study and solution of algebraic differential equations by means of the analysis of an associated algebraic variety (see e.g. [6], [8], [9], [12], [13], [14]); for instance, an algebraic nonautonomous first order ordinary differential equation induces an algebraic surface and the existence, and actual computation, of a general rational solution is derived from a birational parametrization of this surface (see [14]).

Nevertheless, when dealing with parametric representations one needs to guarantee that certain problematic situations do not appear. An specially important difficulty may occur when dealing with parametrizations that are not surjective. That is, let us work with, say, a rational affine variety $X$, and we take a birational affine parametrization $f$ of $X$; in other words, a dominant birational map $f: \mathbb{C}^{r} \rightarrow f\left(\mathbb{C}^{r}\right) \subset X \subset \mathbb{C}^{n}$, and let us assume that $f$ is not surjective, i.e. $f\left(\mathbb{C}^{r}\right) \subsetneq X$. Then, the feasibility of the use of $f$ depends on whether the desired property of the variety, or the information derived from the variety, is only readable from the non-reachable zone $X \backslash f\left(\mathbb{C}^{r}\right)$ of the algebraic variety. Example 1.1., in [20], illustrates the described difficulty for the problem of computing the distance of a point to an algebraic surface. Another example of this situation can be found in Example 1, in [19], 
for computing the intersection of two surfaces using the implicit equation of one of the surfaces, and a parametrization of the other. In [18], Example 1, the authors illustrate the problem of analyzing cross sections in a surface when using a non surjective parametrization. An slightly different version of this commented dificulty can be found in the frame of algebraic differential solutions. Let us say that $y(x, c)$ is the rational general solution of an algebraic non-autonomous first order differential equation $F\left(x, y, y^{\prime}\right)=0$. Then, $\mathcal{P}(x, c)=\left(x, y(x, c), y^{\prime}(x, c)\right)$, where the derivative is with respect to $x$, is a rational surface parametrization of the affine surface $F\left(x_{1}, x_{2}, x_{3}\right)=0$. So, for each specialization of $c, \mathcal{P}(x, c)$ defines an integral rational curve on the surface. Now, observe that the non-surjectivity of $\mathcal{P}$ relates to the existence of rational singular solutions; and example illustrating this phenomenon can be found in [14], Example 7.1.

When the affine complex variety $X$ is a curve, the problem admits a direct solution, in the sense that $X$ can always be parametrized birationally and surjectively. Furthermore, in [1] and [17] one may find algorithms for this purpose. For the case of surfaces, the problem turns to be more complicated. The question has been approached from two different point of views: either providing one surjective birational affine parametrization of $X$ (see e.g. [7], [15] , [19]), or determining finitely many birational affine parametrizations $f_{1}, \ldots, f_{s}$ of $X$ such that the union of their imagines does cover the whole affine surface, that is $\cup_{i=1}^{s} f_{i}\left(\mathbb{C}^{2}\right)=X$ (see e.g. [3], [7], [18], [21], [20]). Nevertheless, the following natural question arises: does there exist a surjective birational affine parametrization for every rational affine surface?

In this paper, we answer this question and we prove that, in general, the answer is no. More precisely, in Theorem 3.1, we describe the intersection of the projective closure of the given affine surface with the plane at infinity under the assumption that the surface can be parametrized surjectively and birationally; in fact, we see that this intersection has to be either smooth or contain at least one rational component. As a consequence, in Example 4.2, we show that the Fermat cubic surface cannot be parametrized surjectively with a birational parametrization.

\section{Preliminaries}

In this section, we recall some basic facts that will be used throughout the paper; we refer the reader to [4], [10] for further details. We also include some consequences whose reference is unknown to us. We work over the complex field $\mathbb{C}$. A variety is an irreducible and reduced projective scheme. We remind the reader that a variety $X$ is normal iff the local ring $\mathcal{O}_{X, x}$ is integrally closed for all $x \in X$. We also recall that any smooth variety is normal.

Some classic results:

Theorem 2.1. [10, Exercise II.3.22(c)] Let $f: X \rightarrow Y$ be a surjective morphism of schemes. Then, the dimension of the general fiber of $f$ is $\operatorname{dim} X-\operatorname{dim} Y$.

Theorem 2.2. [10, Exercise I.3.20] Let $X$ be a quasi projective normal surface. Let $f: X-\rightarrow \mathbb{A}^{N}$ be a rational map, whose indeterminacy locus is finite. Then $f$ is a regular morphism.

Theorem 2.3. [10, Lemma V.5.1] Let $f: X \rightarrow \mathbb{P}^{N}$ be a birational map. If $X$ is normal, the fundamental locus of $f$ has codimension at least 2 in $X$.

Theorem 2.4. [4, Theorem II.7] Let $X$ be a smooth surface. Let $f: X \rightarrow \mathbb{P}^{N}$ be a rational map. Then there exists a commutative diagram

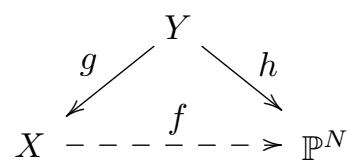


where $g$ is a composite of blowups and $h$ is a morphism.

Corollary 2.5. In the hypotheses of Theorem 2.4, for any fundamental point $P$ of $f, h\left(g^{-1}(P)\right)$ is a connected finite union of rational curves.

Proof. Let $E$ be the connected component $g^{-1}(P)$ of the exceptional divisor. Since $g$ is a composite of blowups, $E$ is a connected union of irreducible curves, all of them isomorphic to $\mathbb{P}^{1}$.

If $h\left(g^{-1}(P)\right)=h(E)$ is not a connected finite union of rational curves, due to the connectedness of $E$ and the fact that it is one dimensional, $h(E)$ must be a single point $Q \in \overline{f(X)} \subset \mathbb{P}^{N}$. Taking an affine neighbourhood $U \subset \mathbb{P}^{N}$ of $Q$, we have that $V=f^{-1}(U) \cup\{P\}$ is neighbourhood of $P$ in $X$. Applying Theorem 2.2 to $\left.f\right|_{V}$, we have that $f$ can be extended to $P$, which contradicts the fact that it is a fundamental point.

Theorem 2.6. [10, Corollary V.5.4 and Theorem V.5.7](Castelnuovo's criterion of contractibility) Let $X$ be a smooth surface and $C$ an irreducible curve in $X$. There exists a smooth surface $Y$ and a birational morphism $f: X \rightarrow Y$ contracting $C$ to a point iff $C \simeq \mathbb{P}^{1}$ and $C^{2}=-1$. In such case, $f$ is the blowup of the point $f(C) \in Y$.

Remark 2.7. Theorem 2.6 says that any birational morphism between nonsingular surfaces is a composite of blowups, each one being the blowup of a closed point.

Regarding blowups, let us recall how they behave with respect to the Picard group:

Theorem 2.8. [10, Exercise II.8.5] Let $f: X \rightarrow Y$ the blowing up of a smooth surface $Y$ at a closed point $y \in Y$. Then $\operatorname{Pic} X \simeq \operatorname{Pic} Y \oplus\langle E\rangle$, where $E$ is the exceptional divisor and the inclusion of Pic $Y$ is given by the pullback $f^{*}$.

Theorem 2.9. (Zariski's Main Theorem, see e.g. [10, Corollary III.11.4]) Let $f: X \rightarrow Y$ be a birational projective morphism between irreducible and reduced varieties. Suppose $Y$ to be normal. Then, for any $y \in Y, f^{-1}(y)$ is connected.

\section{Surjective parametrizations of affine surfaces}

This section is devoted to proving the following result.

Theorem 3.1. Let $f: \mathbb{C}^{2--\rightarrow} \mathbb{C}^{N}$ be a rational map. Let $S$ be the Zariski closure of $f\left(\mathbb{C}^{2}\right)$ in $\mathbb{C}^{N}$, and suppose that $f$ is birational and surjective onto $S$. Let $\bar{S}$ be the Zariski closure of $S$ in $\mathbb{P}^{N}$ and $S_{\infty}=\bar{S}-S$ the hyperplane section at infinity. If $\bar{S}$ is smooth, then $S_{\infty}$ has at least $\rho(\bar{S})$ rational components, where $\rho(\bar{S})$ is the Picard number (i.e. rank of the Picard group) of $S$.

To prove Theorem 3.1, we consider Theorem 2.4 and get the commutative diagram

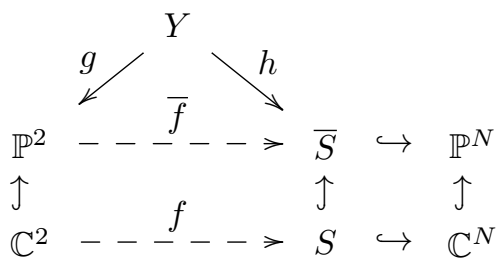


We also establish some more notation. We denote by $F(\bar{f})$ the (finite, by Theorem 2.3) fundamental locus of $\bar{f}$, and by $L_{\infty}=\mathbb{P}^{2} \backslash \mathbb{C}^{2}$ the line at infinity of the plane.

We prove two lemmas before attacking Theorem 3.1.

Lemma 3.2. In the conditions of Theorem 3.1, and with the notation above, then it holds that $h\left(g^{-1}(F(\bar{f})) \subset S_{\infty}\right.$.

Proof. We know by Theorem 2.3 that $F(\bar{f})$ is a finite set. Let $P \in F(\bar{f})$ be one of its elements and let $E=g^{-1}(P)$ be the connected component of the exceptional divisor corresponding to $P$. Let $C=h(E)$. We know by Corollary 2.5 that $C$ is a finite union of rational curves. By Theorem 2.1, any general point $Q \in C$ satisfies that $h^{-1}(Q)$ is zero dimensional (otherwise, $h^{-1}(C)$ would be a surface in $Y$, contradicting the birationality of $f$ ).

On the other hand, Theorem 2.9 implies that $h^{-1}(Q)$ is connected. Therefore, $h^{-1}(Q)$ is a single point $P \in Y$. However, since $Q \in C=h(E)$, this means that $P \in E$, so $Q \notin f\left(\mathbb{C}^{2}\right)$. Since $f$ is surjective onto $S$, this means that $Q \in h(Y) \backslash S=\bar{S} \backslash S=S_{\infty}$. As this happens for general $Q \in C$, we have that $C \subset S_{\infty}$.

This is valid for any $P \in F(\bar{f})$, so the proof is completed.

Lemma 3.3. In the conditions of Theorem 3.1, and with the notation above, it holds that $h\left(g^{-1}\left(L_{\infty}\right)\right) \subset$ $S_{\infty}$

Proof. If $\bar{f}\left(L_{\infty}\right)$ is a curve $C$, by Theorem 2.1, any general point $Q \in C$ satisfies that $h^{-1}(Q)$ is zero dimensional (otherwise, $h^{-1}(C)$ would be a surface in $Y$, contradicting the birationality of $f$ ). On the other hand, Theorem 2.9 implies that $h^{-1}(Q)$ is connected. Therefore, $h^{-1}(Q)$ is a single point $P \in Y$.

However, since $Q \in C=\bar{f}\left(L_{\infty}\right)$, we have that $g\left(h^{-1}(Q)\right)=g(P) \in L_{\infty}$, and then $Q \notin f\left(\mathbb{C}^{2}\right)=S$. This means $Q \in h(Y) \backslash S=\bar{S} \backslash S=S_{\infty}$. The generality of $Q$ means that $\bar{f}\left(L_{\infty}\right) \subset C \subset S_{\infty}$.

Now, consider the case of $f$ contracting $L_{\infty}$ to a point $Q \in S$. Since $S$ is smooth, the proper transform $\widetilde{L_{\infty}}$ of $L_{\infty}$ by $g$ (i.e. $\widetilde{L_{\infty}}$ is the Zariski closure of $g^{-1}\left(L_{\infty}\right)-g^{-1}(F(f))$ ) is a component of the exceptional divisor of $h$. This means that ${\widetilde{L_{\infty}}}^{2}<0$. This is only possible if $g$ blows up at least one point $P \in L_{\infty}$ (i.e. there is a fundamental point of $f$ in $L_{\infty}$ ). Then, by Corollary 2.5 and Lemma 3.2 , we have that $S_{\infty}$ contains $h\left(g^{-1}(P)\right)$, which is one dimensional.

By Theorem $2.9, g^{-1}(P)$ is connected. Therefore, since $P \in L_{\infty}$, we have that $g^{-1}(P) \cup \widetilde{L_{\infty}}$ is connected. This implies that $h\left(g^{-1}(P) \cup \widetilde{L_{\infty}}\right)$ is connected, so $f\left(L_{\infty}\right)=Q \in h\left(g^{-1}(P)\right) \subset S_{\infty}$.

Corollary 3.4. In the hypotheses of Theorem 3.1 and with the notation above, let $C$ be a closed curve in $\mathbb{P}^{2}$ such that $\bar{f}(C)$ consists of a single point $Q \in \bar{S}$. Then $Q \in S_{\infty}$.

Proof. Note that $C \cap L_{\infty}$ is not empty by Bezout's Theorem. Then $C \cup L_{\infty}$ is connected. This means that $h\left(g^{-1}\left(C \cup L_{\infty}\right)\right)$ is connected. Since $h\left(g^{-1}\left(L_{\infty}\right)\right)$ is always one dimensional (in the proof of Lemma 3.3, we see that, if $L_{\infty}$ is contracted by $\bar{f}$, then $L_{\infty} \cap F(f)$ is not empty), this means that $Q \in h\left(g^{-1}\left(L_{\infty}\right)\right)$, so $Q \in S_{\infty}$ by Lemma 3.3. 
Proof. (of Theorem 3.1) Let $\rho(Y)$ be the Picard number of $Y$. Since $\rho\left(\mathbb{P}^{2}\right)=1$, we have that $g$ is a composition of $\rho(Y)-1$ blowing ups by Theorem 2.8. This means that the exceptional divisor $E$ consists of $\rho(Y)-1$ rational components. On the other side, by Theorem 2.8 and Remark 2.7, $h$ contracts exactly $\rho(Y)-\rho(\bar{S})$ irreducible rational curves. This means that $h\left(g^{-1}\left(L_{\infty}\right) \cup E\right)$, being the image by $h$ of $\rho(Y)$ rational curves consists of at least $\rho(\bar{S})$ rational curves. By Lemmas 3.2 and 3.3, we have that $h\left(g^{-1}\left(L_{\infty}\right) \cup E\right) \subset h\left(g^{-1}\left(L_{\infty} \cup F(f)\right)\right) \subset S_{\infty}$, so we have at least $\rho(\bar{S})$ rational curves in $S_{\infty}$.

Corollary 3.5. Let $S \subset \mathbb{A}^{N}$ be a closed surface. Let $\bar{S}$ be its Zariski closure in $\mathbb{P}^{N}$. Suppose that $\bar{S}$ is smooth and that none of the components of $S_{\infty}=\bar{S}-S$ is rational. Then there does not exist a birational surjective parametrization $f: \mathbb{A}^{2} \rightarrow S$.

Proof. For any surface $S, \rho(\bar{S})$ is at least one.

\section{Examples and nonexistence results}

The next example illustrates that the conditions given by Theorem 3.1 are sharp. We find a family of examples where just one rational component at the infinity is enough to have a surjective parametrizations from $\mathbb{C}^{2}$.

Example 4.1. Consider the d-th Veronese embedding $v_{d}: \mathbb{P}^{2} \rightarrow \mathbb{P}^{N}$, where $N=\frac{(d+2)(d+1)}{2}$, given by all degree $d$ monomials: $v_{d}\left(x_{0}: x_{1}: x_{2}\right)=\left(x_{0}^{d}: x_{0}^{d-1} x_{1}: \ldots: x_{2}^{d}\right)$. Let $C \subset \mathbb{P}^{2}$ be a curve of degree $d-1$ and $L_{\infty}$ be the line at infinity $x_{0}=0$. Then the ideal of $C \cup L_{\infty}$ is given by a homogeneous polynomial of degree $d$. This means that $v_{d}\left(C \cup L_{\infty}\right)$ is the intersection of $\widetilde{S}=v_{d}\left(\mathbb{P}^{2}\right)$ with a hyperplane $H$. Therefore, if we compose $v_{d}$ with the suitable automorphism of $\mathbb{P}^{N}$ that takes $H$ to the hyperplane at infinity, we get a parametrization $f$ of the image $\bar{S}$ such that $S_{\infty}=f\left(C \cup L_{\infty}\right)$. This means that $S=\bar{S} \backslash S_{\infty}$ is covered by $\mathbb{P} \backslash\left(C \cup L_{\infty}\right)=\mathbb{C}^{2} \backslash C$, and $f_{\mathbb{C}^{2} \backslash C}$ is an isomorphism.

The next example is one of the main motivations of this paper: finding examples of rational affine surfaces that do not admit birational surjective parametrizations.

Example 4.2. Consider $\bar{S}$ to be a smooth cubic surface. It is well known that $\bar{S}$ is isomorphic to $\mathbb{P}^{2}$ blown up at 6 generral points. Then $\rho(\bar{S})=7$. On the other side, due to the degree, $S_{\infty}$ has at most three components. Then, by Theorem 3.1, it is impossible to give a birational surjective parametrization of the complement in $\bar{S}$ of any hyperplane section.

Example 4.3. Consider $\bar{S}$ to be a smooth quadric hypersurface. There are only two possibilities for $S_{\infty}$. If $S_{\infty}$ has singularities, then it must consist of two lines. Then the structure of $\bar{S}$ as $\mathbb{P}^{1} \times \mathbb{P}^{1}$ gives the affine part the structure of $\mathbb{C}^{1} \times \mathbb{C}^{1} \simeq \mathbb{C}^{2}$. We would have the well-known parametrization of the paraboloid. However, if the curve at infinity is nonsingular, then it is an irreducible conic. Since $\rho(\bar{S})=\rho\left(\mathbb{P}^{1} \times \mathbb{P}^{1}\right)=2$, by Theorem 3.1, there does not exist a birational surjective parametrization of $\bar{S} \cap \mathbb{A}^{3}$.

The following example shows that Theorem 3.1 is not a characterization:

Example 4.4. Let $\bar{S}$ be $\mathbb{P}^{1} \times \mathbb{P}^{1}$. Let $C$ and $C^{\prime}$ be two curves in $\bar{S}$ of types $(2,1)$ and $(1,2)$ respectively (regarding $\bar{S}$ as a quadric, $C$ and $C^{\prime}$ would be twisted cubics). Since $C+C^{\prime}$ is of type $(3,3)$, it is a very ample divisor. This means that we can consider $\bar{S} \subset \mathbb{P}^{15}$ with $S_{\infty}=C+C^{\prime}$. 
Now suppose there exists $f: \mathbb{A}^{2} \rightarrow S=\bar{S}-S_{\infty}$ birational and surjective. Consider the extension of the inverse $f^{-1}$ to the complement of a finite subset of $\bar{S}$. Such map can only contract curves in $S_{\infty}$ (if $\bar{f}^{-1}\left(C^{\prime \prime}\right)$ is a point $P \in \mathbb{P}^{2}$, then $P \in F(f)$ and, by Lemma 3.2, we have that $C^{\prime \prime} \subset S_{\infty}$ ).

Looking at the proof of Theorem 3.1, at least one among $C$ and $C^{\prime}$ corresponds to a fundamental point $P$ of $f$. Let us say that $C \subset h\left(g^{-1}(P)\right)$. Then, $g$ contracts the proper transform $\widetilde{C}$ of $C$ by $h$ to $P$. This means that $\widetilde{C}$ is a component of the exceptional divisor $E$ of $g$, so it has negative self-intersection. If $g$ is the composition $g_{s} g_{s-1} \cdots g_{1} g_{0}$ of blowups, then $h$ must be a composition $h_{s} h_{s-1} \cdots h_{2} h_{1}$ of blowing downs. This means that $E$ consists of $s+1$ rational components. Since $h\left(E+\widetilde{L_{\infty}}\right) \subset S_{\infty}=C+C^{\prime}$, we get that $h$ contracts exactly s components of $E+\widetilde{L_{\infty}}$. Since $h$ factors as exactly s blowups, this means that $h$ does not contract any curve outside $E+\widetilde{L_{\infty}}$. Then there are two possibilities:

- If $h$ covers $C^{\prime}$ with $\widetilde{L_{\infty}}$ and $C$ with $\widetilde{C}$. Then $h$ contracts all of $E-\widetilde{C}$ to points. This implies that $C \cap C^{\prime}$ consists of just one point, due to the nature of blowing up.

- If $h$ contracts $\widetilde{L_{\infty}}$, then $h$ contracts all components of $E$ but two, used to cover $C$ and $C^{\prime}$. This also implies that $C \cap C^{\prime}$ consists of just one point, due to the nature of blowing up.

However, choosing general $(2,1)$ and $(1,2)$ curves for $C$ and $C^{\prime}$ one obtains that $C \cap C^{\prime}$ consists of 5 different points, leading to contradiction. Therefore, $S$ cannot be covered by a birational surjective parametrization despite it satisfies the necessary condition of Theorem 3.1

Acknowledgments. Authors partially supported by Ministerio de Economía y Competitividad and the European Regional Development Fund (ERDF), under the Project MTM2014-54141-P; and by Junta de Extremadura and FEDER funds (group FQM024).

The authors would like to thank Jeremy Blanc for his suggestion to use the Picard number of the surface.

\section{References}

[1] Carlos Andradas and Tomás Recio. Plotting missing points and branches of real parametric curves. Appl. Algebra Engrg. Comm. Comput., 18(1-2):107-126, 2007.

[2] Chandrajit L. Bajaj, editor. Algebraic geometry and its applications. Springer-Verlag, New York, 1994. Papers from the conference held in honor of Shreeram Shankar Abhyankar at Purdue University, West Lafayette, Indiana, June 1-4, 1990.

[3] Chandrajit L. Bajaj, A. V. Royappa. Finite representations of real parametric curves and surfaces. Internat. J. Comput. Geom. Appl. 5 (1995), no. 3, 313-326

[4] Arnaud Beauville. Complex algebraic surfaces, volume 34 of London Mathematical Society Student Texts. Cambridge University Press, Cambridge, second edition, 1996. Translated from the 1978 French original by R. Barlow, with assistance from N. I. Shepherd-Barron and M. Reid.

[5] Gerald Farin, Josef Hoschek, and Myung-Soo Kim, editors. Handbook of computer aided geometric design. North-Holland, Amsterdam, 2002.

[6] R. Feng, Xiao Shan Gao Gao. A polynomial time algorithm for finding rational general solutions of first order autonomous ODEs. J. Symbolic Comput. 41 (7) (2006) 739-762. 
[7] Xiao Shan Gao and Shang-Ching Chou. On the normal parameterization of curves and surfaces. Internat. J. Comput. Geom. Appl., 1(2):125-136, 1991.

[8] Georg Grasegger. Radical solutions of algebraic ordinary differential equations, in: K. Nabeshima (Ed.), Proceedings of the 2014 International Symposium on Symbolic and Algebraic Computation (ISSAC), ACM Press, New York, 2014, pp. 217-223.

[9] Georg Grasegger, Alberto Lastra, J. Rafael Sendra, Franz Winkler. A solution method for autonomous first-order algebraic partial differential equations. Journal of Computational and Applied Mathematics 300 (2016) 119-133.

[10] Robin Hartshorne. Algebraic geometry. Springer-Verlag, New York-Heidelberg, 1977. Graduate Texts in Mathematics, No. 52.

[11] Josef Hoschek and Dieter Lasser. Fundamentals of computer aided geometric design. A K Peters, Ltd., Wellesley, MA, 1993. Translated from the 1992 German edition by Larry L. Schumaker.

[12] Evelyne Hubert. The general solution of an ordinary differential equation, in: Y.N. Lakshman (Ed.), Proceedings of the 1996 International Symposium on Symbolic and Algebraic Computation (ISSAC), ACM Press, New York, 1996, pp. 189-195.

[13] L.X.Chau Ngô, J. Rafael Sendra, F. Winkler. Birational transformations on algebraic ordinary differential equations. J. Comput. Appl. Math. 286 (2015) 114-127.

[14] L.X.Chau Ngô, Franz Winkler. Rational general solutions of first order non-autonomous parametrizable ODEs. J. Symbolic Comput. 45 (12) (2010) 1426-1441.

[15] Sonia Pérez-Díaz, J. Rafael Sendra, Carlos Villarino. A First Approach Towards Normal Parametrizations of Algebraic Surfaces. International Journal of Algebra and Computation Vol. 20, No. 8 (2010) 977-990

[16] Thomas W. Sederberg. Applications to computer aided geometric design. In Applications of computational algebraic geometry (San Diego, CA, 1997), volume 53 of Proc. Sympos. Appl. Math., pages 67-89. Amer. Math. Soc., Providence, RI, 1998.

[17] J. Rafael Sendra. Normal parametrizations of algebraic plane curves. J. Symbolic Comput., 33(6):863-885, 2002.

[18] J. Rafael Sendra, David Sevilla, and Carlos Villarino. Covering of surfaces parametrized without projective base points. In ISSAC 2014-Proceedings of the 39th International Symposium on Symbolic and Algebraic Computation, pages 375-380. ACM, New York, 2014.

[19] J. Rafael Sendra, David Sevilla, and Carlos Villarino. Some results on the surjectivity of surface parametrizations. In Computer algebra and polynomials, volume 8942 of Lecture Notes in Comput. Sci., pages 192-203. Springer, Cham, 2015.

[20] J. Rafael Sendra, David Sevilla, and Carlos Villarino. Covering rational ruled surfaces. Math. Comp. Volume 86, Number 308, November 2017, Pages 2861-2875.

[21] J. Rafael Sendra, Carlos Villarino, and David Sevilla. Missing sets in rational parametrizations of surfaces of revolution. Comput.-Aided Des., 66:55-61, 2015.

Jorge Caravantes, Research Group GVP

Dpto. de Álgebra, Universidad Complutense de Madrid 
Plaza de Ciencias 3, 28040 Madrid, Spain

Email: jcaravan@mat.ucm.es

J. Rafael Sendra, Research Group ASYNACS

Dpto. de Física y Matemáticas, Universidad de Alcalá

28871 Alcalá de Henares (Madrid), Spain

E-mail: Rafael.sendra@uah.es

David Sevilla, Research group GADAC

Centro U. de Mérida, Universidad de Extremadura

Av. Santa Teresa de Jornet 38, 06800 Mérida (Badajoz), Spain

E-mail: sevillad@unex.es

Carlos Villarino, Research Group ASYNACS

Dpto. de Física y Matemáticas, Universidad de Alcalá

28871 Alcalá de Henares (Madrid), Spain

E-mail: Carlos.villarino@uah.es 